- There have been concerns expressed about metal root canal posts and cores with regard to corrosion, risk of root fracture and aesthetic outcome. Therefore, alternative non-metallic posts and cores have been introduced into dentistry.

- This study examines the retention of some prefabricated root canal posts made of a variety of materials that have been recently introduced into dentistry.

- This study gives information about the retention in vitro of prefabricated root canal posts made of a variety of materials. To assess their suitability for use as an alternative to individually cast posts controlled clinical studies are indicated.

\title{
Retention of prefabricated and individually cast root canal posts in vitro
}

\author{
S-O. Hedlund, ${ }_{1}^{1}$ N. G. Johansson ${ }^{2}$ and G. Sjögren ${ }^{3}$
}

Objectives The aim of the study was to evaluate the retention of prefabricated root canal posts made of a variety of materials that have recently been introduced into dentistry.

Materials and methods The posts studied were CosmoPost, Composipost carbon fibres, Composipost Æsstheti-Plus, Composipost Light-Post and Para Post Fiber White. The posts were luted in extracted human premolars and the cores were built up with the resin composites recommended by the manufacturers. The retention of individually cast gold alloy posts luted with zinc-phosphate cement were used as reference. A universal testing machine was used to determine the retention of each cemented post. Data were compared using ANOVA supplemented with Fisher's PLSD at a significance level of $p<0.05$. Results Only the CosmoPost system exhibited retention values that were significantly lower than for the conventionally cast gold alloy posts luted with zinc-phosphate cement. The force necessary to loosen the CosmoPost specimens was significantly less than that needed to loosen the Composipost Æstheti-Plus $(p<0.05)$ and the Composipost Light-Post systems $(p<0.001)$. The force necessary to loosen the Para Post Fiber White specimens was significantly less than for the Composipost Light-Post system $(p<0.01)$. Other combinations did not differ significantly $(p>0.05)$.

Conclusions When zirconium oxide ceramic posts are luted with resin composites the bonding between the ceramic and resin composite seems to be weak. Further studies are thus necessary to achieve improved retention of all-ceramic root canal posts.

\section{INTRODUCTION}

Individually cast metal posts and cores are a commonly used technique to improve the retention of dental crowns and bridges on nonvital teeth. As an alternative to the individually cast posts,

\footnotetext{
${ }_{1 *}$ Former Assistant Professor. Department of Dental Materials Science, Faculty of Medicine and Odontology, Umeå University ${ }^{2}$ Former Assistant Professor. Department of Dental Materials Science, Faculty of Medicine and Odontology, Umeå University ${ }^{3}$ Associate Professor, Department of Dental Materials Science, Faculty of Medicine and Odontology, Umeå University

${ }^{*}$ Correspondence to: Dr. Sven-Olof Hedlund, Dental Materials Science, Faculty of Medicine and Odontology Umeå University SE-901 87 Umeå, Sweden

Email:Sven-Olof.Hedlund@odont.umu.se
}

\section{Refereed paper}

Received 21.05.02; Accepted 21.11.02

doi:10.1038/sj.bdj.4810405

๑ British Dental Journal 2003; 195: 155-158 prefabricated metal posts provided with glass ionomer cement or composite cores, are used. However, the risk of root fracture is substantial when endodontically treated teeth are provided with metal posts. ${ }^{1,2}$ It has been suggested that the difference between the elastic modulus of dentin and post material is a source of stress for the root structures, ie the more rigid post resists the force and transfers the stress to the less rigid tooth substance thus causing failure of the tooth structure. ${ }^{3}$ Corrosion has been proposed as a cause of fracture of the metallic posts. ${ }^{4}$ Another disadvantage is that opaque metal posts and cores may shine through semi-translucent all-ceramic crowns and impart a greyish colour to all-ceramic crowns thus influencing the aesthetic outcome of the restoration. ${ }^{5}$ Metal posts may also shine through thin cervical areas and influence the appearance of the gingival tissue and both root discolouration and corrosion products in the gingival tissues have been reported in connection with teeth provided with nonprecious metal posts. ${ }^{6,7}$ In addition, because of the public scare about possible adverse side effects of dental metallic restorations, the demand for non-metallic dental restorations has increased during the past decades. Consequently, alternative non-metallic posts and cores have been introduced into dentistry during the past decade, eg carbon fibre posts in an epoxy fibre matrix, quartz fibre posts, carbon fibre posts covered with quartz fibres and all-ceramic posts.,9 These posts are intended to be adhesively luted into the root canal using resin composites and the core is subsequently built up with a resin composite. Retention of the posts is believed to be essential for the longevity of restorations placed on endodontically treated teeth provided with intra-coronal posts. Therefore, the aim of the present study was to compare the force needed to loosen root canal posts fabricated using a variety of materials and provided with a core of resin composite.

\section{METHODS AND MATERIALS}

Seventy sound, caries-free, single-rooted extracted human premolars were used for this study. The extracted premolars had been stored in $0.5 \%$ benzalconium chloride. The crowns of the premolars were removed $\sim 1 \mathrm{~mm}$ coronal to the cementoenamel junction using a diamond disk with water coolant and a low ratio handpiece, with the teeth fixed in a stand. The section was made perpendicular to the long axis of the tooth. Thereafter the premolars were randomly divided into 7 groups of 10 , one group of 10 as prepared for conventionally cast gold alloy posts, the second group 
for CosmoPost (Ivoclar AG, Schaan, Lichtenstein, batch no: 549403 ), the third for carbon fibres Composipost NO 3 (RTD, Saint-Egrève, France, batch no: 3L N0010A), the fourth for white quartz-fibres Composipost NO 3 Æstheti-Plus (RTD, batch no: 3 SQ 0009A), the fifth for white translucent-quartz fibres Composipost NO 3 Light-Post (RTD, batch no: 3 SR 0007A), the sixth for white translucent-quartz fibres Composipost NO 3 Light-Post (RTD, batch no: 3 SR 0007A) with a funnel-shaped inlet of the postholes, and the last group for Para Post Fiber White (Coltene/Whaledent, Wellingford, CT., USA, batch no: MT-28423). The diameter of the CosmoPost was $1.7 \mathrm{~mm}$ and the diameter of the Composipost was $2.1 \mathrm{~mm}$ (coronal section) and $1.4 \mathrm{~mm}$ (apical section), respectively. The diameter of the Para Post Fiber White was $1.5 \mathrm{~mm}$.

The root canal preparation of the premolars intended for conventionally cast gold alloy posts was performed with a dental bur with a $6^{\circ}$ taper (JS Davis Reamer No; 13, Svenska Dental Instrument, Stockholm, Sweden) and a low ratio handpiece. The length of the root canal preparation was $8 \mathrm{~mm}$. The manufacturers' instructions were followed for the root canal preparation of the CosmoPost, Composipost and Para Post Fiber White specimens. In addition, to study the effect of a wide inlet for the post-holes for the Composipost Light-Post, the coronal section of the root canal preparation was funnel-shaped using a dental bur with a $45^{\circ}$ taper and a low ratio handpiece. Using the lost-wax technique the conventionally cast gold alloy posts and cores were cast in JS Sjödings C-gold (JS Sjödings AB, Kista, Stockholm). The cores were shaped so that there was a $\sim 1.2-1.5 \mathrm{~mm}$ wide shoulder all around the cast core.

The coronal surface and the root canal preparations of all the premolars were thereafter rinsed with pumice using Young screw type polishers (Young Dental Manufacturing, Earth City, MO., USA) and inter-dental brushes, rinsed with water, and finally gently dried with air. The conventionally cast gold alloy posts were cemented with a zinc-phosphate cement (Phospha CEM IC Capsule, Vivadent, Schaan, Lichtenstein. Batch no: C0 1698). The CosmoPost was luted with Syntac (Vivadent, primer batch no: D 13804, adhesive batch no: D 09384) and a dual-cured resin composite, Varioloink II (Vivadent, base batch no: D 07406, catalyst batch no: C 15156). The Para Post Fiber White was luted with a chemically-cured resin composite, ParaPost Cement (Coltene/Whaldent, batch no: KG 778). The Composipost fibre posts were cemented with a dual-cured resin cement, Cement-It (Jeneric/Pentron Inc. Wellington, CT, USA, batches no: 33114 for the base and 33113 for the catalyst) after the canal walls and coronal surfaces were etched with a $37 \%$ phosphoric acid etching gel (Jeneric/Pentron Inc., batch no: 35088) for 15 seconds, rinsed with water, and finally dried with air and absorbent paper points, in accordance with the manufacturer's instruction. The primer/adhesive system used for Composipost was Bond 1 Primer/Adhesive (Jeneric/Pentron Inc.,Wellingford, CT., USA, batch no: 34560). When the Composipost specimens were luted, double layers of the bonding agent were applied on the post, the canal walls, and the coronal surface of the premolars in accordance with the manufacturer's instruction. Excess luting agent was removed with Quick-Sticks (Dentonova AB, Stockholm, Sweden). The luting agent, ie Cement-It, used to cement the translucent quartz-fibres Composipost Light-Post was also polymerised by means of a halogen lamp (Norlite, Germany) for 20 seconds. All the Composipost, CosmoPost and Para Post Fiber White were then cut with a diamond disk and a handpiece at a distance of $\sim 5 \mathrm{~mm}$ from the coronal surface of the premolars.

In order to position the premolars so that the vertical load to be applied on the apical surface of the posts would be as parallel as possible to the direction of the long-axis of the teeth when they were mounted and loaded in a testing machine, moulds of hole for the coronal section of the posts, copper rings (diameter 12 polyvinyl-chloride (Gehr Plastics Inc., Boothwyn, PA, USA) with a

$\mathrm{mm}$, height $7 \mathrm{~mm}$ ), and a 2-component methacrylate ester (Protemp, ESPE Dental AG, Seefeldt, Germany, batch no: B 090) were used. The copper ring was placed in the polyvinyl-chloride mould, then the coronal part of the posts were inserted into the hole in the mould until the coronal surface of the premolars rested against the mould. Thereafter, the copper ring was filled with the 2-component methacrylate ester (Protemp) surrounding the coronal section of the premolar (Fig. 1). After polymerisation of the 2-component methacrylate ester (Protemp) excess material on the coronal surface of the teeth was removed with a dental diamond bur after the premolars had been removed from the polyvinyl chloride mould.

After this the CosmoPost and Composipost posts were provided with cores of a dual-cured resin composite (Build-It Core Material, Jeneric/Pentron Inc., batches no: 33690 for the base and 33681 for the catalyst). For ParaPost Fibre White the dual-cured resin cement, ParaCore, was used (Coltene/Whaledent, batch no: KE 080). The resin composites for the cores were polymerised by means of a halogen lamp (Norlite) for 1 minute. Using a dental cylindrical diamond bur and an air turbine handpiece with water coolant a $\sim 1.2-1.5 \mathrm{~mm}$ wide shoulder was then shaped all around the core (Fig. 2). Thereafter the apical section of all premolars was removed, $\sim 1 \mathrm{~mm}$ from the apical end of the cemented posts, with a cylindrical dental diamond bur and an air turbine handpiece with water coolant. By means of a dental bur, $1 \mathrm{~mm}$ diameter, a hole was then drilled into the apical end of the posts. Subsequently, all the premolars with the cemented posts and cores were stored in a saline solution for one week at $37^{\circ} \mathrm{C}$ in the dark.

One week after cementation a universal testing machine (Alwetron TCT 5/10, Lorentzon and Wettre, Spånga, Sweden) was used to determine the retention of each cemented post. The premolars with the posts were placed in the testing machine with the coronal part down and with the $\sim 1.2-1.5 \mathrm{~mm}$ wide shoulder around the cores resting on a metal plate and with the cores free to move downwards. A brass mould around the coronal section of the teeth that was provided with the copper ring and the 2-component methacrylate ester (Protemp) supported the premolars (Fig. 2). By means of a steel stylus with a diameter of $1 \mathrm{~mm}$ (Fig. 2) and using a crosshead speed of $0.5 \mathrm{~mm} / \mathrm{min}$ a vertical load was applied to the

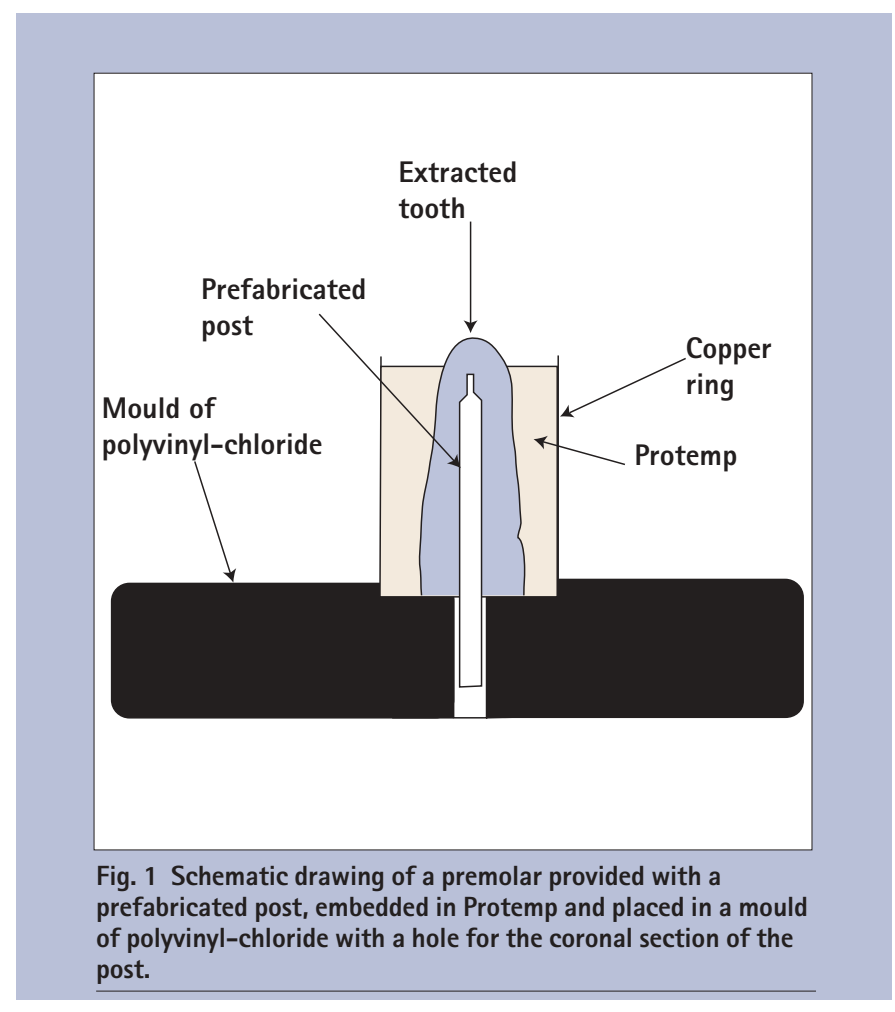




\begin{tabular}{|c|c|c|c|}
\hline Specimens & Mean (N) & 95\% confidence interval & $p$-value \\
\hline $\begin{array}{l}\text { Conventionally cast } \\
\text { gold alloy luted with } \\
\text { zinc-phosphate cement }\end{array}$ & 179 & $139.15,218.85$ & \\
\hline CosmoPost & 89 & $111.87,60.13$ & 0.047 \\
\hline $\begin{array}{l}\text { Composipost } \\
\text { carbon fibres }\end{array}$ & 169 & $229.76,108.10$ & 0.837 \\
\hline Composipost Æstheti-Plus & 201 & $280.71,121.29$ & 0.646 \\
\hline Composipost Light-Post & 259 & $352.43,165.57$ & 0.097 \\
\hline $\begin{array}{l}\text { Composipost Light-Post } \\
\text { with funnel-shaped inlet } \\
\text { of the post holes }\end{array}$ & 261 & $367.49,154.51$ & 0.090 \\
\hline Para Post Fiber White ${ }^{\S}$ & 108 & $147.85,68.15$ & 0.154 \\
\hline
\end{tabular}

apical end of the cemented post (Fig. 3). The force necessary to loosen the post was automatically recorded at when the force was $1 \%$ below the highest level recorded during the test before fracture was detected and the testing interrupted. To ensure that the post/core had loosened and that the steel stylus had loaded the apical end of the posts during the testing the borders around the cores and the apical end of the teeth were examined with a light microscope at $12 \mathrm{x}$ magnification.

\section{Statistical analysis}

The values were statistically analyzed using analysis of variance (ANOVA) supplemented with Fisher's PLSD at a significance level of $p<0.05$.

\section{RESULTS}

The mean values and 95\% confidence levels $(n=10)$ of the force needed to loosen the root canal posts are presented in Table 1 . In the Para Post Fiber White group $n=9$ because one specimen was accidentally lost. Only the CosmoPost system exhibited retention values that were significantly lower than for the conventionally cast gold alloy posts luted with zinc-phosphate cement $(p<0.05)$. In addition, the force needed to loosen the CosmoPost specimens was significantly lower than for the Composipost Æstheti-Plus ( $\mathrm{p}=$ 0.02), the Composipost Light-Post $(p=0.0004)$, and the Composipost Light-Post with funnel-shaped inlet $(p=0.0004)$. The force necessary to loosen the Para Post Fiber White specimens was significantly lower than for the Composipost Light-Post $(p=0.003)$ and the Composipost Light-Post with funnel-shaped inlet $(\mathrm{p}=$ 0.003). Other combinations were not significantly different ( $p>$ 0.05).

\section{DISCUSSION}

Determination of the retention of intra-coronal posts has been used to compare various types of root canal posts. Usually the posts that exhibit the greater values are considered to be less likely to loosen when subjected to stress. ${ }^{10,11}$ In most studies a tensile force has been used to determine the values required to remove the post from the root canal. ${ }^{\text {eg } 10,12}$ In the current study, pressure was applied on the apical end of the luted posts because the aim was to determine the retentive force of various types of root canal posts after they had been provided with a core of resin composite. Since it was difficult to avoid damaging the core when the upper part of the specimen was fixed in a testing machine and also to minimise unwanted lateral forces this specific experimental set-up was selected.

The root canal posts evaluated in the current study consist of a variety of materials. The carbon fibre posts, ie Composipost, are made from continuous, unidirectional carbon fibres, diameter $\sim 8$ $\mu \mathrm{m}$, in an epoxy resin matrix. ${ }^{8}$ According to the manufacturer the Composipost Æstheti-Plus and the Composipost Light-Post consist of quartz fibres in an epoxy resin matrix. CosmoPost is, according to the manufacturer, fabricated from stabilised zirconium ceramic $\left(\mathrm{ZrO}_{2}\right)$ and the surfaces of these posts are sandblasted during the manufacturing process. The Para Post Fiber White consists, according to the manufacturer, of glass fibres, resin and filler. The reason for also evaluating the retention of a conventionally cast gold alloy post luted with zinc-phosphate cement in the present

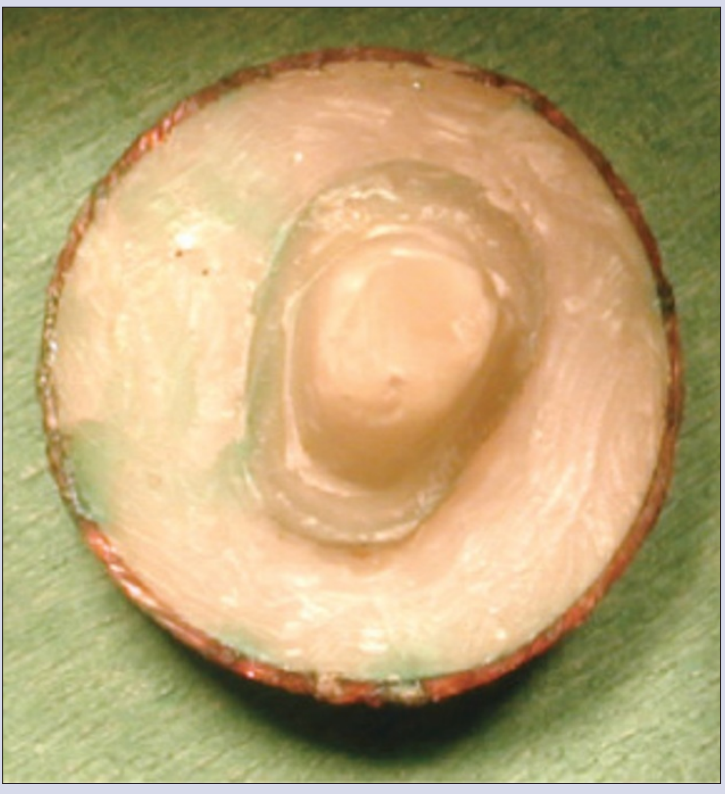

Fig. 2 Premolar embedded in Protemp and provided with a prefabricated post and a resin composite core before post dislodgement.

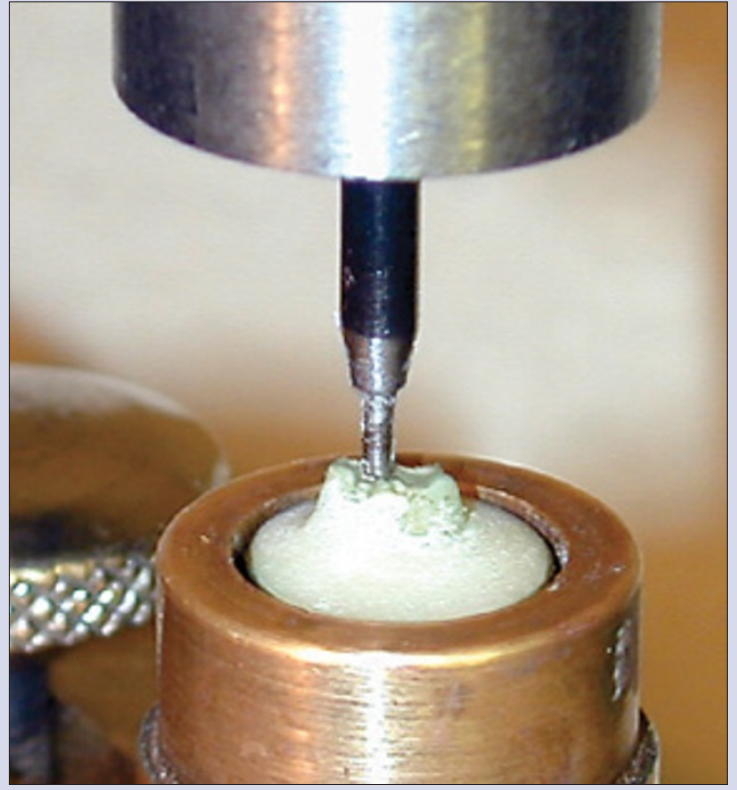

Fig. 3 Test apparatus used for retention test. 
study was to facilitate the comparison of the values obtained for the new post systems evaluated in this study with a well-known and frequently used technique.

Not only does the material of the root canal posts affect the retention of the restorations but the properties of the luting agents may also influence the bonding strength. In the present study the resin composites and the luting techniques used were in accordance with the manufacturers' instructions. Therefore, the luting agent and the core materials used differ among the specimens studied. The differences obtained between the forces necessary to loosen the prefabricated posts may, thus, have been influenced by the properties of the luting agent and the core materials. However, since the manufacturers recommended the use of those materials it seemed reasonable and correct to use them when their root canal post systems were seated and when the cores were built up. Comparison of the values obtained for the force needed to loosen the specimens reveals that only the CosmoPost system showed a significantly lower retention compared with the conventionally cast gold alloy posts. One possible reason for the lower values obtained for the CosmoPost specimens is that, although the surfaces of the posts had already been sandblasted with aluminium oxide when delivered from the manufacturer, the bonding of the resin composite to the ceramic posts seemed to be insufficient. Visual inspection of the surfaces of the posts after the CosmoPost specimens were removed from the root canal, showed that there were no remnants of the luting agent on those posts. This implies an adhesive failure at the interface between the cement and the ceramic. In an earlier study of the retentive strength of another ceramic post system made of yttrium oxide stabilised zirconium oxide (Cerapost) luted using a resin composite, it was reported that sandblasting the posts using aluminium oxide prior to cementing did not significantly improve retention. ${ }^{12}$ In addition, some of the sandblasted posts fractured during the retention test. ${ }^{12}$ Further studies are thus necessary to achieve improved retention of all-ceramic root canal posts. Regarding the Composipost carbon fibre, the Composipost Light-Post, and Para Post Fiber White, visual inspection of the specimens after they were removed from the root canals showed that more cement was retained than on the CosmoPost but there were areas free of cements, implying that the mode of failure was a combination of adhesive and cohesive failure at the ceramiccement interface. The conventionally cast gold alloy posts and the Composipost Æstheti-Plus retained a large amount of cement, indicating adhesive failure at the cement-root canal interface.

To study the effect of the design of the coronal inlet of the post hole on the retention, two different coronal shapes of the root canal preparation for the Composipost Light-Post specimens were evaluated: a funnel-shaped inlet and an inlet shaped by the recommended dental bur. However, no significant difference between the two types of preparation was observed. Thus, a wider inlet resulting in a larger luting agent surface against the tooth substance did not significantly influence the retention of the root canal posts (Table 1).

In the current study there is a wide range of measurements in the standard deviations obtained for some of the specimens. However, comparison of the values reported in some earlier studies dealing with the retention of root canal posts using extracted human teeth reveals that there are also wide ranges in those measurements. ${ }^{\text {eg } 10,12,13}$ One possible explanation for the wide ranges is that the studies used extracted human teeth. The size and shape of the root canals may differ and/or the texture and properties of the inner surfaces of the root canals can differ among the teeth used. This, however, is also the case in clinical situations.

In a survey of the literature, few articles address the retention of the prefabricated posts studied in the current study. Therefore, comparisons were made with some similar prefabricated posts. In an earlier study of the retention of another type of a carbon fibre root canal post (Endoposts), the force needed to loosen those posts cemented with a resin cement was $134(\mathrm{SD}=$ 52) N. ${ }^{14}$ Purton et al. ${ }^{12}$ reported values between $118(\mathrm{SD}=21) \mathrm{N}$ and $190(\mathrm{SD}=95) \mathrm{N}$ for the retentive strength of ceramic posts made of zirconium oxide (Cerapost) luted with a resin composite. In another study of this type of ceramic post (Cerapost) luted with a resin composite the retention was $104(\mathrm{SD}=35) \mathrm{N} .{ }^{15} \mathrm{In}$ the current study the force was recorded when it was $1 \%$ below the highest level recorded during the test, whereas in the other studies the force required to remove the post from the root was recorded. This may be one reason for the differences between the values obtained in the current study and in some earlier studies. In addition, in the current study the posts were provided with cores and thus partly retained to the coronal surface of the teeth. However, the possibility can not be excluded that stresses caused by the polymerising shrinking of the core could pull the post in a coronal direction and weaken the bonding of the post to the luting agent and/or the root canal. Clinically posts and cores are subjected to conditions that are difficult to simulate in vitro. Controlled clinical studies of the prefabricated posts evaluated in the current study are therefore indicated in order to assess their suitability for use as an alternative to individually cast posts.

1. LovendahI PE, Nicholls J I. Pin-retained amalgam cores vs. cast-gold dowel-cores. J Prosthet Dent 1977: 38: 507-514.

2. Tamse A. latrogenic vertical root fractures in endodontically treated teeth. Endod Dent Traumatol 1988; 4: 190-196.

3. Mannoci F, Innocenti M, Ferrari M, Watson T F. Confocal and scanning electron microscopic study of teeth restored with fiber posts, metal posts, and composite resins. J Endod 1999; 25: 789-794.

4. Silness J, Gustavsen F, Hunsbeth J. Distribution of corrosion products in teeth restored with metal crowns retained by stainless steel posts. Acta Odontol Scand 1979; 37: 317-321.

5. Siber C, Thiel N. Spinell/Luminary porcelain: natural light optics for anterior crowns. Quintessence Dent Technol 1996; 19: 43-49.

6. Wirz J, Christ R. In vitro study of the occurrence of screw and pin corrosion in dental prosthesis structures [in German]. Schweiz Monatsschr Zahnheilkd 1982; 92: 408-428.

7. Meyenberg K. Dental esthetics - a European perspective. J Esthet Dent 1994; 6: 274-281.

8. Mannocci F, Ferrari M, Watson TF. Intermittent loading of teeth restored using quartz fiber, carbon -quartz fiber, and zirconium dioxide ceramic root canal posts. J Adhes Dent 1999; 1: 153-158.

9. Koutayas S O, Kern M. All-ceramic posts and cores: the state of the art. Quintessence Int 1999; 30: 383-392

10. Cohen $B \mid$, Musikant B L, Deutsch AS. Comparison of retentive properties of four post systems. J Prosthet Dent 1992; 68: 264-268.

11. Cohen B I, Pagnillo M K, Newman I, Musikant B L, Deutsch A S. Retention of three endodontic posts cemented with five dental cements. J Prosthet Dent 1998; 79: 520-525

12. Purton D G, Love R M, Chandler N P. Rigidity and retention of ceramic root canal posts. Oper Dent 2000; 25: 223-227.

13. Love R M, Purton D G. Retention of posts with resin, glass ionomer and hybrid cements. J Dent 1998; 26: 599-602.

14. Purton D G, Love R M. Rigidity and retention of carbon fibre versus stainless stee root canal posts. Int Endod J 1996:29:262-265.

15. Cohen B I, Pagnillo M K, Newman I, Musikant B L, Deutsch A S. Retention of four endodontic posts cemented with composite resin. Gen Dent 2000; 48: 320-324. 